\title{
A Simple Approximation of the Aggregate Interference From a Cluster of Many Interferers With Correlated Shadowing
}

\author{
Sebastian S. Szyszkowicz, Member, IEEE, and Halim Yanikomeroglu, Senior Member, IEEE
}

\begin{abstract}
We examine the statistical distribution of the interference produced by a cluster of many co-channel interferers: e.g., a sensor network, or a city full of active wireless devices and access points. We consider a clustered interferer layout and analyze the interference as experienced at a given point outside (and not immediately near to) the interferer area. We model the propagation paths as experiencing power law attenuation and lognormal correlated shadowing, with pairwise correlations depending on the relative positions of the two interferers. It has already been shown in literature that adding correlation to the shadowing model can give significantly different, and more realistic, results. Our solution is mostly analytical, with only a small amount of numerical integration required. Whereas both simulation and other analytical methods become very computationally demanding as the number of interferers grows, our method's complexity is independent of the number of interferers, and its precision actually improves when their number increases.
\end{abstract}

Index Terms-Sum of lognormals, correlated shadowing, interference analysis.

\section{INTRODUCTION}

$\mathbf{S}$ CARCE wireless spectrum is in ever-increasing demand, due to a multitude of emerging rate-hungry applications. Satisfying this demand will require an increased spatial reuse of the wireless channel. While in the past wireless systems were often noise-limited, or experienced only a few co-channel interferers, we expect future systems to often be significantly interference-limited, with potentially hundreds of co-channel interferers. It is therefore of interest to study the statistics of the total interference power coming from many interferers; in [1], we examine some properties of this total interference power, focusing on rapid simulation algorithms for a large number

Manuscript received September 17, 2013; revised January 13, 2014; accepted March 30, 2014. Date of publication April 14, 2014; date of current version August 8, 2014. This work was supported in part by the National Sciences and Engineering Research Council (NSERC) of Canada. This work has appeared, in part, in the proceedings of the IEEE Wireless Communications and Networking Conference, 2008 Las Vegas, NV, USA, under the title Analysis of Interference from Large Clusters as Modeled by the Sum of Many Correlated Lognormals. The associate editor coordinating the review of this paper and approving it for publication was R. Zhang.

S. S. Szyszkowicz is with the School of Electrical Engineering and Computer Science, University of Ottawa, Ottawa, ON K1N 6N5, Canada (e-mail: sz@sce.carleton.ca).

H. Yanikomeroglu is with the Department of Systems and Computer Engineering, Carleton University, Ottawa, ON K1S 5B6, Canada (e-mail: halim@ sce.carleton.ca).

Color versions of one or more of the figures in this paper are available online at http://ieeexplore.ieee.org.

Digital Object Identifier 10.1109/TWC.2014.2317709 of interferers. Whereas shadowing correlation is of secondary importance in scenarios with only a few interferers (such as in classical cellular networks), in [1] we demonstrate that shadowing correlation plays a significant role in the statistical behavior of the total interference power from a large number of interferers, and cannot realistically be neglected (as it often had been before [2]-[8]).

The sum interference power from many transmitters has received significant interest in recent years [7]-[9], including scenarios where transmitters are grouped in one or multiple clusters [10], [11]. Important emerging applications include machine-to-machine (M2M) networks [12] (including wireless sensor networks [9]-[11]), ad-hoc networks [10], [11], and large cities with thousands of devices simultaneously transmitting in unlicensed bands (e.g., WiFi, Bluetooth [11], [13]-[15]); this results in many possible scenarios where even thousands of small simultaneous transmitters distributed over a certain area collectively cause non-negligible interference to receivers located outside that area (sometimes even tens of kilometers away [14], [15]).

Despite this growing importance of modeling the interference from many devices, the channel models used in the analysis literature are inadequate to the task (most likely due to the analytical difficulty of the problem): lognormal shadowing is not always considered, and furthermore, to the best of our knowledge, only our work in [1] considers position-dependent correlation among shadowing paths. Work on interference with correlated shadowing has been relatively scarce. The study of the distribution of the sum of correlated lognormal random variables (RVs) has received some interest [16]-[21] (see also some references therein). This type of work, however, presupposes deterministic interferer positions, which are needed to obtain the pairwise correlation coefficients; it is not immediately evident how to extend those methods to a random interferer placement. Furthermore, there is some difficulty in scaling these methods, due to their computational complexity and a possible deterioration in accuracy as the number of interferers increases. Recent works [22], [23] examining sum interference with correlated shadowing consider only a small number of interferers (at most 6) and assume constant pairwise correlation, with the constant value chosen more or less arbitrarily. But actually, there exist many models for shadowing correlation [24], and in this work we allow for any reasonable shadowing correlation function. We study the case of hundreds of individual interferers, and allow their positions to be random according 
to some spatial distribution. Indeed, it may be simpler and more credible to describe a large number of positions statistically rather than deterministically.

In this paper, we continue the work in [1] with roughly the same problem statement, and provide a very simple and accurate analytical approximation to the sum interference power distribution for a particular subset of possible interferer layouts, specifically what we call a cluster, i.e., from the vantage point of the receiver, all the interference signals arrive from within a narrow angle spread. In this case, the total interference power is shown to follow an approximately lognormal distribution, as long as the number of interferers is large enough. A simple analytical solution is interesting in that it gives insight into the effect of various system and channel parameters on the total interference power distribution; also, correlation makes the problem computationally very expensive for a large number of shadowing paths. This paper complements our work in [1], which is a partly-simulation-based method that we recommend for non-cluster geometries to which the approximations presented here do not apply.

In Section II, we describe the physical problem and its mathematical model, after having introduced some necessary mathematical concepts. The problem statement is a slightly simplified version of the one we used in [1]. We derive a simple lognormal approximation to the total interference power distribution in Section III, with details of the derivation in the Appendices. We demonstrate the accuracy of our approximation against Monte Carlo simulations in Section IV. In Section V, we conclude and give some ideas on how to extend our method to more detailed system and channel models.

\section{Problem Statement}

Our problem statement is novel for its combination of shadowing with spatially-dependent correlation, random interferer positions according to any spatial distribution, and a large number of interferers. We used a similar problem statement, with minor variations, in [1], [25], [26].

\section{A. Mathematical Tools}

We first introduce some mathematical concepts that will be needed for describing and analyzing our problem.

The theory of exchangeable RVs was introduced by de Finetti [27]-[29]. Intuitively, a set of exchangeable RVs is simply one for which the index assignment is arbitrary, i.e., all permutations of the RVs have the same joint distribution.

Definition 1: A finite set of RVs $\left\{X_{i}\right\}_{i=1}^{N}$ is said to be exchangeable if the vectors $\left[X_{1}, X_{2}, \ldots, X_{N}\right]$ and $\left[X_{p(1)}, X_{p(2)}\right.$, $\left.\ldots, X_{p(N)}\right]$ have the same joint distribution for any permutation $p(i)$ on the vector $[1,2, \ldots, N]$.

Theorem 1: (Hewitt-Savage Generalization of de Finetti's Theorem [30]). For any exchangeable set $\left\{X_{i}\right\}_{i=1}^{N}$, there exists a single RV $Z$ such that all $X_{i}$ 's are conditionally independent on $Z$. Equivalently, there exists a set of independent and identically distributed (iid) $\operatorname{RVs}\left\{Y_{i}\right\}_{i=1}^{N}$ such that, for all $i, X_{i}$ can be written as a function of $Z$ and $Y_{i}$.

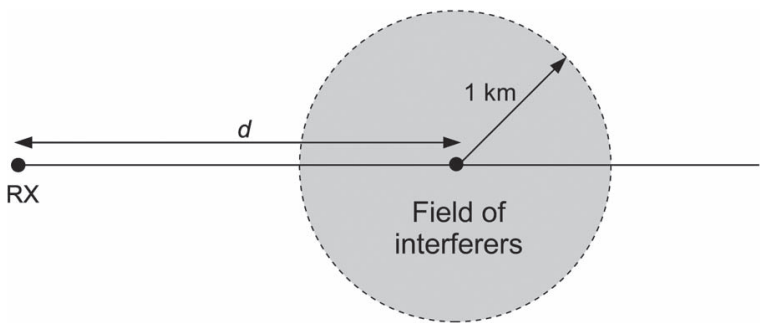

Fig. 1. Example of a clustered layout, used for the simulations in this paper. The interferers are distributed uniformly and independently over the circular region of radius $1 \mathrm{~km}$, and the victim receiver $\mathrm{RX}$ is located at $d \gg 1 \mathrm{~km}$ from its center.

Definition 2: Consider a set $\left\{X_{i}\right\}_{i=1}^{N}$ of (jointly) Gaussian RVs. We say that the RVs $\left\{e^{X_{i}}\right\}_{i=1}^{N}$ are jointly lognormal.

Definition 3: A sequence $X_{1}, X_{2}, \ldots, X_{N}$ of RVs with cumulative distribution functions (cdfs) $F_{1}(x), F_{2}(x), \ldots, F_{N}(x)$ converges in distribution [31] to an RV $X$ with cdf $F(x)$ if

$$
\lim _{N \rightarrow \infty} F_{N}(x)=F(x), \quad \forall x \in \mathbb{R} .
$$

We then write: $X_{N} \stackrel{\mathcal{D}}{\longrightarrow} X$ as $N \rightarrow \infty$.

\section{B. System Model}

We consider $N$ interferers, randomly distributed according to a density function $g(\vec{r})$, such that the victim receiver RX experiencing the interference is defined to be located at the origin. This can model interferer layouts of any shape, such as those found in literature: circles or rings [1, and references therein], hexagonal or circular cellular layouts [2], [23], [32][34], or even detailed densities of urban areas [14], [15], whereas networks of infinite size such considered in [7], [8] are merely mathematical simplifications of finite networks.

The locations $\vec{r}_{i}$ of the interferers are iid; we write $r_{i}=\left\|\vec{r}_{i}\right\|$. We assume each interferer transmits with equal constant power.

We will further assume a clustered layout of the interferer density $g(\vec{r})$, meaning that:

1) Most if not all of $g(\vec{r})$ is located far enough away from the RX (in its far field).

2) The RX sees most of $g(\vec{r})$ inside a fairly narrow angle, so that the average correlation between shadowing paths is high.

Fig. 1 gives an illustrative cluster layout scenario. The distance scale is an example only, as the problem can be scaled to be smaller or larger. Notably, in [14], [15], whole cities with thousands of WiFi devices are located tens of kilometers away from a weather radar [14] or television receivers [15] to which they cause interference.

\section{Channel Model}

The channel model is described by the distance-dependent pathloss and by the spatially correlated shadowing. We do not consider small-scale fading.

We assume power law pathloss with exponent $\beta$. A constant power law can be justified as long as the interferers are not 
located too close to the RX; e.g., in [35] the pathloss is modeled as approximately proportional to $r_{i}^{-4}$ for $r_{i}>200 \mathrm{~m}$.

We also assume lognormal shadowing, with constant $\mathrm{dB}$ spread $\sigma_{\mathrm{s}}$. Again, the constant $\mathrm{dB}$ spread can be justified if the interferers are not located very close to the RX; e.g., in [36] the shadowing spread is approximately $10 \mathrm{~dB}$ for $r_{i}>200 \mathrm{~m}$.

The interference power received at the RX produced by a single interferer $i$ is then a RV, which can be written

$$
I_{i}=K r_{i}^{-\beta} e^{\lambda S_{i}}
$$

where $\lambda=0.1 \ln 10, S_{i}$ is a zero-mean Gaussian RV with variance $\sigma_{\mathrm{s}}^{2}$, and $K$ is a constant common to all interferers that includes factors such as interferer transmit power, pathloss at a reference distance, transmit and receive antenna gains, etc.

This, however, is not a full characterization of the interference powers $I_{i}$, since they are in fact correlated through the correlation of the shadowing terms $S_{i}$. We construct a vector $\vec{S}=\left[S_{i}\right]_{i=1}^{N}$ that is (jointly) Gaussian when conditioned on $\vec{r}_{1}, \ldots, \vec{r}_{N}$. The vector is fully described statistically by the quantities

$$
\begin{aligned}
\mathbb{E}\left\{S_{i}\right\} & =0, \\
\mathbb{E}\left\{S_{i}^{2}\right\} & =\sigma_{\mathrm{s}}^{2}, \\
\mathbb{E}\left\{S_{i} S_{j} \mid \vec{r}_{i}, \vec{r}_{j}\right\} & =\sigma_{\mathrm{s}}^{2} h\left(\vec{r}_{i}, \vec{r}_{j}\right), \quad i \neq j,
\end{aligned}
$$

where $h\left(\vec{r}_{i}, \vec{r}_{j}\right)$ is the shadowing correlation model, which must satisfy the following conditions:

1) $-1 \leq h\left(\vec{r}_{i}, \vec{r}_{j}\right) \leq 1$.

2) $h\left(\vec{r}_{i}, \vec{r}_{i}\right)=1$.

3) $h\left(\vec{r}_{i}, \vec{r}_{j}\right)=h\left(\vec{r}_{j}, \vec{r}_{i}\right)$.

4) The correlation matrix of $\vec{S}$ must always be positive semidefinite (psd). This can be guaranteed only by certain functions $h\left(\vec{r}_{i}, \vec{r}_{j}\right)$ that have the "positive semidefinite" property [24], [37].

It should also be remarked that, once the conditioning on the set $\left\{\vec{r}_{i}\right\}$ is removed, the set $\left\{S_{i}\right\}$ is no longer jointly Gaussian, though each $S_{i}$ is still individually Gaussian.

We examined shadowing correlation models in [24], where we justified the choice of the following model (which is inspired by [38]):

$$
h\left(\vec{r}_{i}, \vec{r}_{j}\right)=\max \left\{1-\theta / \theta_{0}, 0\right\} \cdot \max \left\{1-R / R_{0}, 0\right\},
$$

where $\theta=\left|\angle \vec{r}_{i}-\angle \vec{r}_{j}\right| \in\left[0^{\circ}, 180^{\circ}\right]$ is the angle of arrival separation, and $R=(10 / \ln 10)\left|\ln r_{i}-\ln r_{j}\right|$ is the ratio (in $\mathrm{dB}$ ) of the propagation path lengths. The model has two tunable parameters: $0^{\circ}<\theta_{0} \leq 180^{\circ}$ and $R_{0}>0$.

We choose this model among many others for the following reasons:

1) We show in [24] that this model always yields psd correlation matrices. This is not the case for several of the existing models [24].

\footnotetext{
${ }^{1}$ This time, a property of shadowing correlation models. Positive semidefinite functions $h\left(\vec{r}_{i}, \vec{r}_{j}\right)$ are those that always yield psd correlation matrices, for any $N$.
}

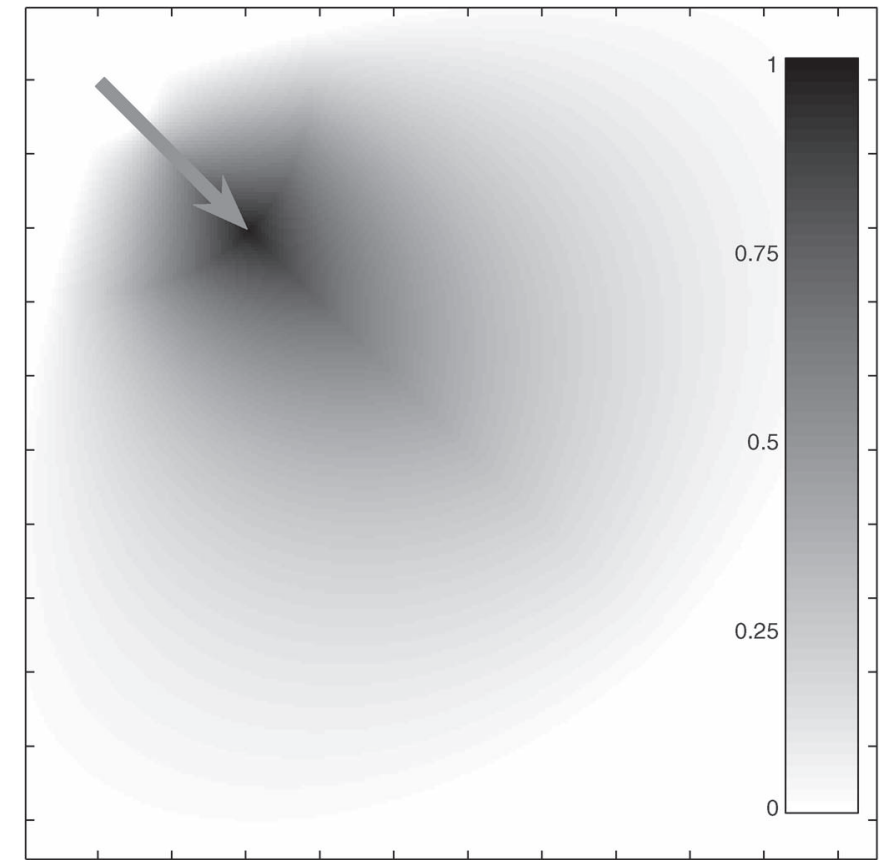

Fig. 2. Shadowing correlation function (3) with $\theta_{0}=60^{\circ}$ and $R_{0}=6 \mathrm{~dB}$ : the correlation coefficient between two shadowed paths beginning at the base of the arrow. One path ends at the tip of the arrow, while the other is located anywhere on the plane. The correlation function is invariant under rotation and scaling.

2) Furthermore, we argue in [24] that, from among all models that always give psd correlation matrices, this model seems most physically plausible. In particular, we contrast this model with those expressible in the form $h\left(\vec{r}_{i}, \vec{r}_{j}\right)=f\left(\left\|\vec{r}_{i}-\vec{r}_{j}\right\|\right)$, notably with $f(x)=e^{-x / d_{0}}$ [39]. We argue that these models are difficult to reconcile with the propagation arguments for correlation in shadowing given in [32], [38], [40].

3) The selected model has two tunable parameters, and can therefore approximate a wide range of correlation models with reasonable accuracy, as shown in [38].

4) The mathematical form of this model lends itself particularly well to fast simulation using shadowing fields, as we demonstrate in [1].

We choose the values $\theta_{0}=60^{\circ}$ and $R_{0}=6 \mathrm{~dB}$, which are realistic values based on [38]. This particular model is illustrated in Fig. 2. Again, this is only one possible model, and our analysis is independent of the form of $h\left(\vec{r}_{i}, \vec{r}_{j}\right)$.

Adding correlation to shadowing represents an important increase in the accuracy of the physical model, in the difficulty of the analysis, and in the computational cost of simulation. The addition of correlation significantly broadens the distribution of $I$ by increasing its variance, as we have shown in [1] through analysis and simulation examples.

\section{Sum Interference}

It is often assumed [16], [41] that the total interference power at the RX is the sum of the individual interference powers; this 
is justified by the incoherent addition of interfering signals. The total interference power is then given by

$$
I=\sum_{i=1}^{N} I_{i}=K \sum_{i=1}^{N} r_{i}^{-\beta} e^{S_{i}} .
$$

It is this RV $I$ that we wish to study.

In [1], we study the moments of $I$ and some of its asymptotic properties as $N \rightarrow \infty$. We also showed how $I$ can be rapidly simulated for large $N$ with good accuracy. In this paper,we give a lognormal approximation to the distribution of $I$, which we find to be accurate when $N$ is large and the interferers are in a clustered layout.

\section{ANALYSIS}

It would be possible to use direct moment matching of the first two moments of $I$ with those of a lognormal RV, as done in, e.g., [17], [34]. However, the moments of $I$ have complicated integral expressions [1], and moment matching tends to bias the approximation towards the upper tail of the cdf [41]. Instead, we develop a two-step approach to solving the problem. In the first step, we show the convergence in distribution of the sum of exchangeable joint lognormal (SEJLN) RVs to a lognormal distribution. In the second step, we argue that the interference problem is similar (but not identical) to the SEJLN problem, and we match the parameters of the two problems through logarithmic cross-moment matching (in [41], we see that matching the moments of the logarithm of a sum of lognormal RVs tends to approximate the middle of the distribution well). Our final result is a simple expression for the parameters of the lognormal distribution approximating that of $I$, requiring only a minimum of numerical integration involving only the functions $g(\vec{r})$ and $h\left(\vec{r}_{i}, \vec{r}_{j}\right)$.

\section{A. Limit Theorem on the Sum of Exchangeable Joint Lognormal RVs}

Let $\left[V_{i}\right]_{i=1}^{N}$ be a vector of $N$ jointly Gaussian RVs, each with the same mean $\mu$, same variance $\sigma^{2} \neq 0$, and each pair with the same correlation coefficient $0<\rho \leq 1$. Now let

$$
V=\sum_{i=1}^{N} e^{V_{i}}
$$

We say that $V$ follows a SEJLN distribution with parameters $\mu$, $\sigma^{2}, \rho$, and $N$.

Theorem 2: Let $V$ follow a SEJLN distribution. Then, as $N \rightarrow \infty$, the quantity $V / N$ tends in distribution to a lognormal $\mathrm{RV}$ with parameters $\left(m_{\infty}, s_{\infty}^{2}\right)$ given by

$$
m_{\infty}=\mu+\frac{1}{2}(1-\rho) \sigma^{2}, \quad s_{\infty}^{2}=\rho \sigma^{2} .
$$

We prove this theorem in [18]. Appendix A gives a similar proof, but with a simpler decomposition. This theorem is also extended to a more general case in [21].

\section{B. Lognormal Approximation of the SEJLN RV for Large $N$}

Given that the SEJLN distribution is lognormal in the limit as $N \rightarrow \infty$, it would also be interesting to examine its behavior for moderate values of $N$. We reason that, since for both $N=$ 1 and $N \rightarrow \infty$, the SEJLN distribution is lognormal, then it might be approximately lognormal for intermediate values of $N$, particularly large ones.

Matching of the first two moments of $V$ with those of a lognormal RV with parameters $m_{V}$ and $s_{V}^{2}$ yields [18]

$$
\begin{aligned}
m_{V} & =\mu+\frac{3}{2} \ln N-\frac{1}{2} \ln \left(1+(N-1) e^{(\rho-1) \sigma^{2}}\right), \\
s_{V}^{2} & =\sigma^{2}-\ln N+\ln \left(1+(N-1) e^{(\rho-1) \sigma^{2}}\right) .
\end{aligned}
$$

At this point one may note that the construction of the SEJLN distribution is based on equal means, variances, and correlation coefficients of the underlying Gaussian RVs. It may therefore appear that this problem has little to do with finding the distribution of $I$ in (4), where the means and correlation coefficients are distinct. Nevertheless, we proceed to show how this ancillary mathematical result can be used to find an approximate solution for the distribution of $I$.

\section{Mapping the SEJLN Problem Onto the Sum Interference Problem}

Let us now make some observations about the set $\left\{e^{V_{i}}\right\}$ :

1) It is exchangeable. In fact, for any set of $N$ exchangeable RVs, each pair must have the same correlation coefficient $\rho \in[-1 /(N-1), 1][29]$.

2) It is "augmentable", i.e., adding new terms by increasing $N$ does not change the distribution of the previous terms, because we fix $\rho$ not to change with $N$.

3) The elements are each individually lognormal.

4) They are also jointly lognormal.

Now, let us look at the same properties for the set $\left\{I_{i}\right\}$. We have, respectively:

1) As argued before, it is exchangeable.

2) It is also augmentable. Indeed, adding new interferers should not affect the joint distribution of the previous interferers' powers.

3) The elements are approximately lognormal, as long as the shadowing spread is large enough.

4) Given the previous point, it may be impossible to consider jointness if the individual $I_{i}$ are not even lognormal. However, even the jointness of the terms $S_{i}$ is not guaranteed, since their correlation matrix is random (a function of $\left\{\vec{r}_{i}\right\}$ ), and therefore we cannot even know if the $I_{i}$ 's are approximately jointly lognormal in any sense.

We observe that the two problems are identical in the first two properties, but not in the two others. The last two properties may or may not be fulfilled with any good accuracy, and it is in these two approximations that the inaccuracy of our method lies. We observe in simulations in [25], [26] and herein that the 
approximation is particularly good for a clustered layout. This can be explained as follows:

1) A clustered layout admits only a limited relative spread for $r_{i}$, and thus limits the $\log$-variance of $r_{i}^{-\beta}$ : it follows that the shape of $I_{i}$ is dominated by that of $e^{\lambda S_{i}}$, and that therefore $I_{i}$ is approximately lognormal.

2) A clustered layout imposes a high expected value of the correlation between any two $S_{i}$ 's, due to the angular proximity of the propagation paths. This high correlation may affect the jointness of the $S_{i}$ 's. This can be intuitively explained thus: the higher the correlation terms, the less "freedom" for the joint distribution of the RV and thus perforce the joint distribution must be close to that of a jointly lognormal set of RVs. In particular, if all the correlation coefficients are identically 1 , the joint distribution would admit no freedom at all: all the RVs would be equal and, of course, jointly lognormal.

\section{Cross-Moment Matching and Solution}

The similarities between the SEJLN problem and the interference problem lead us to attempt to match the two, and to use the limit result from the SEJLN to construct an approximate limit result for the distribution of $I$.

Apart from the number of summands, which is $N$ in both problems, the SEJLN distribution is characterized by the three parameters $\mu, \sigma$, and $\rho$. We therefore want three equations to solve for these parameters, and we therefore match the logarithmic moments $\mathbb{E}\left\{\ln I_{i}\right\}$ and $\mathbb{E}\left\{\ln ^{2} I_{i}\right\}$ and the logarithmic crossmoment $\mathbb{E}\left\{\ln I_{i} \ln I_{j}\right\}, i \neq j$. Other moment choices would also be possible, but these were the most natural ones; a crossmoment is essential to capture the correlation between the $I_{i}$ terms.

The details of the moment matching are given in Appendix B. We find that $I$ may be approximated by a lognormal RV with parameters

$$
\begin{aligned}
m_{I} & =\frac{3}{2} \ln N+\ln K-\beta G_{1}-\frac{1}{2} k, \\
s_{I}^{2} & =\lambda^{2} \sigma_{\mathrm{s}}^{2}-\ln N+\beta^{2}\left(G_{2}-G_{1}^{2}\right)+k,
\end{aligned}
$$

where

$$
k=\ln \left(1+(N-1) e^{\left(\lambda^{2} \sigma_{\mathrm{s}}^{2}\left(G_{\mathrm{cor}}-1\right)-\beta^{2}\left(G_{2}-G_{1}^{2}\right)\right)}\right) .
$$

For large $N$, this is well approximated by

$$
\begin{aligned}
m_{I} & \approx \ln K N+\frac{1}{2} \beta^{2}\left(G_{2}-G_{1}^{2}\right)-\beta G_{1}+\frac{1}{2} \lambda^{2} \sigma_{\mathrm{s}}^{2}\left(1-G_{\mathrm{cor}}\right), \\
s_{I}^{2} & \approx \lambda^{2} \sigma_{\mathrm{s}}^{2} G_{\mathrm{cor}} .
\end{aligned}
$$

$G_{n}$ and $G_{\text {cor }}$ are to be found by numerical integration of the functions $g(\vec{r})$ and $h\left(\vec{r}_{i}, \vec{r}_{j}\right)$ :

$$
\begin{aligned}
G_{n} & =\iint_{\mathcal{A}_{g}} \ln ^{n}(r) \mathrm{d} \vec{r}, \\
G_{\text {cor }} & =\iint_{\mathcal{A}_{g}} \iint_{\mathcal{A}_{g}} h\left(\vec{r}_{1}, \vec{r}_{2}\right) g\left(\vec{r}_{1}\right) g\left(\vec{r}_{2}\right) \mathrm{d} \vec{r}_{1} \mathrm{~d} \vec{r}_{2},
\end{aligned}
$$

where $\mathcal{A}_{g}$ is the area over which $g(\vec{r})$ is non-zero. These integrals are well-behaved and can be well approximated by a Riemann sum (trapezoidal rule) with a moderate number of terms. $G_{n}$ and $G_{\text {cor }}$ are termed "geometric coefficients", and are an idea borrowed from [33], [34]. It should be noted that this numerical integration is inevitable, due to the infinite variety of possibilities for the expression of the functions $g(\vec{r})$ and $h\left(\vec{r}_{i}, \vec{r}_{j}\right)$. However, our solution is elegant in that it separates these integrals from the scalar quantities $\beta, \sigma_{\mathrm{s}}$, and $N$, in which it is purely analytical. For this reason, and the brevity of the final equations, we believe this solution to be the simplest possible for this level of modeling accuracy.

\section{E. Interpretation of the Analytical Result}

Several previous works [5], [7], [8] involving a large number of interferers neglect shadowing correlation, and the Central Limit Theorem is invoked to predict a Gaussian distribution of power. Our work shows that, when increasing the level of realism to include shadowing correlation, then $I$ can be approximated by a lognormal distribution (with non-vanishing spread).

We may interpret our result through Theorem 1: indeed, it would seem that each shadowing term is approximately composed of a lognormal component with spread $\sqrt{G_{\text {cor }}} \sigma_{\mathrm{s}}$ common to every path, as well as an additional lognormal term that is iid for every path. As the paths add, the power of the common term grows as $N^{2}$, while the power of the sum of the iid terms only grows as $N$ and becomes more and more negligible, relatively speaking. This is analogous to what happens in our proof in Appendix A. This interpretation is also consistent with the physical basis for correlation in shadowing given in [24], [32], [38], [40].

From the analytical expressions (8) and (10), one can directly observe the effect of the problem parameters on the distribution of $I$.

Of particular interest is that only one scalar quantity depends on the shadowing correlation function $h\left(\vec{r}_{i}, \vec{r}_{j}\right)$, namely $G_{\mathrm{cor}}$. This leads us to formulate a guideline: while it is very important to include correlation in our model, only the scalar $G_{\text {cor }}$ is actually needed in the analytical approximation. $G_{\text {cor }}$ can be interpreted as the "average" correlation coefficient between any two shadowing terms, without conditioning on the positions $\left\{\vec{r}_{i}\right\}$. Indeed

$$
\mathbb{E}\left\{S_{i} S_{j}\right\}=\sigma_{\mathrm{s}}^{2} G_{\text {cor }}, \quad \forall i, \forall j \neq i
$$

which can be contrasted with the conditional correlation in (2). This scalar $G_{\text {cor }}$ is much easier to measure and estimate than the entire four-dimensional function $h\left(\vec{r}_{i}, \vec{r}_{j}\right)$. In general, we think this kind of analysis can point the way for engineers performing channel measurements to know what statistics are actually relevant to a particular problem, thus greatly reducing measurement efforts for particular scenarios.

Our result is based on a limit theorem [18] and therefore is particularly well-suited for large $N$. It is less accurate for small $N$, for which Monte Carlo simulations or recent results on sums of correlated lognormal RVs [19], [20], [42] may sometimes 
be more appropriate. These last approaches, however, do not scale well with $N$ (neither in computational cost nor, perhaps, in accuracy), and therefore our method complements those approaches nicely. We see in the next section that our approximation can predict the results of Monte Carlo simulations well.

\section{Simulation}

We now compare our proposed approximate solution to Monte Carlo simulations. We begin by describing the classical approach for computing the distribution of $I$, including shadowing correlation.

\section{A. Simulation Algorithm}

The computational complexity of the Monte Carlo simulation of $I$ principally resides in the generating of the correlated shadowing values in $\vec{S}$.

The natural approach is first to generate the set of interferer positions $\left\{\vec{r}_{i}\right\}$, and then the $N \times N$ correlation matrix $\mathbf{H}$ with entries $H_{i, j}=h\left(\vec{r}_{i}, \vec{r}_{j}\right)$. We then need to find a factorization of $\mathbf{H}$ that satisfies

$$
\mathbf{H}=\mathbf{C}^{\mathrm{T}} \mathbf{C} .
$$

This is usually done through Cholesky factorization [38], [43]. The existence of a solution ${ }^{2}$ for $\mathbf{C}$ can be guaranteed when the correlation model is "positive semidefinite" [24]. Finally, we need to generate a vector $\vec{Z}=\left[Z_{i}\right]_{i=1}^{N}$ of independent standard Gaussian random variables. We can then generate $\vec{S}$ from

$$
\vec{S}=\sigma_{\mathrm{s}} \vec{Z} \mathbf{C} .
$$

The above algorithm is exact to within numerical precision and has a computational time complexity of $\mathcal{O}\left(N^{3}\right)$ [43], and a memory complexity of $\mathcal{O}\left(N^{2}\right)$. In [1], we demonstrated an approximate algorithm for generating the sets $\left\{S_{i}\right\}$ and $\left\{I_{i}\right\}$, with complexity of $\mathcal{O}(N)$ and even of $\mathcal{O}(1)$ for high enough $N$. For the simulations in this section, we still use the classical Cholesky factorization approach, which is feasible on a personal computer for $N=1000$, but which requires several hours to execute per cdf. Each cdf is generated from 1000000 independent Monte Carlo trials of $I$ (with new values of both $\left\{\vec{r}_{i}\right\}$ and $\vec{S}$ for each trial), and is plotted on "lognormal paper" [41], where all, and only, lognormal cdf appear as straight lines.

\section{B. Simulation Setup}

The simulation parameters are chosen as follows:

- $K=1$,

- $\beta=3.5$,

- $\sigma_{\mathrm{s}}=9(\mathrm{~dB})$,

- $g(\vec{r})$ as in Fig. 1 with $d \in[1.1,6](\mathrm{km})$,

- $h\left(\vec{r}_{i}, \vec{r}_{j}\right)$ as in (3) with $\theta_{0}=60^{\circ}, R_{0}=6(\mathrm{~dB})$,

- $N$ variable up to 1000 .

\footnotetext{
${ }^{2}$ There will generally be many solutions for $\mathbf{C}$, only one of which is the (upper diagonal) Cholesky factorization. However, any solution for $\mathbf{C}$ is equally valid for generating $\vec{S}$.
}

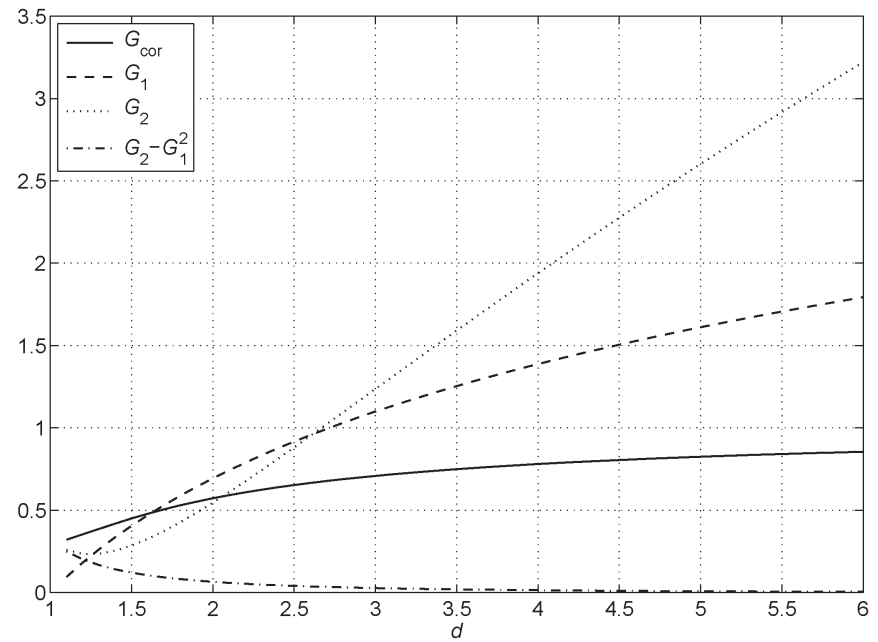

Fig. 3. Geometric parameters as a function of $d$, for the layout in Fig. 1 and correlation function in Fig. 2.

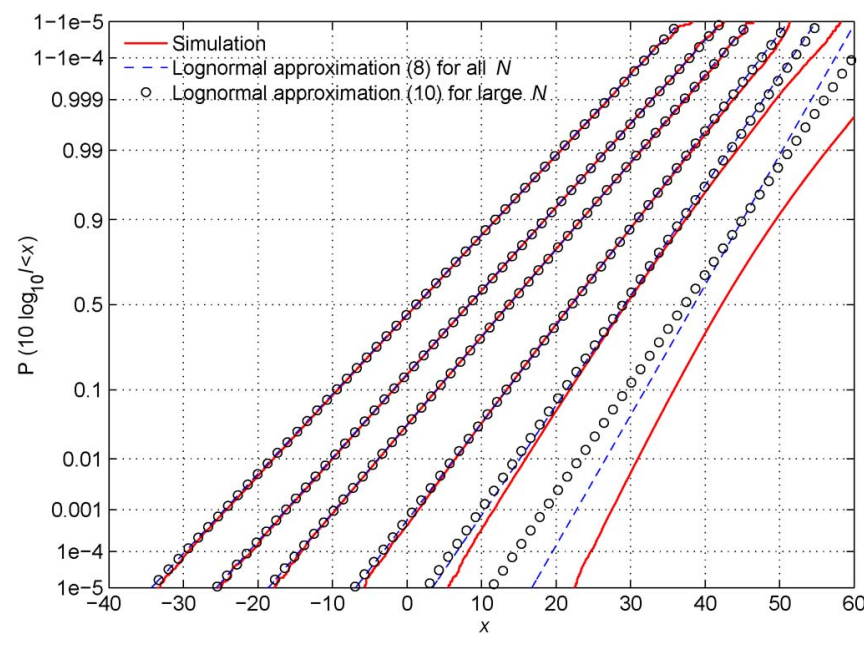

Fig. 4. Distribution of the total interference power $I$ as plotted on "lognormal paper" [41]. The interference is measured at distances of $d=6,4,3,2,1.5$, $1.1 \mathrm{~km}$ (from left to right) from the center of the interferer field, with $N=500$ interferers.

The resulting geometric coefficients, found through numerical Riemann integration of (11), are given in Fig. 3 for various values of $d$. The statistical behavior of $I$ is illustrated in Fig. 4, with the RX placed at various distances from the interferer field, and in Fig. 5, with a variable number of interferers distributed over the same area.

\section{Interpretation of Simulation Results}

The qualitative behavior of the geometric coefficients as a function of $d$ (Fig. 3) lends itself to simple interpretation. First, the quantity $G_{\mathrm{cor}}$, which is the correlation coefficient between any $S_{i}$ and $S_{j}, i \neq j$, increases with $d$. Indeed, as the RX moves farther away from the interferer field, this field is seen by the RX at a smaller angle and relative distance spread. It follows, not only for the correlation model in (3), but for many correlation models (studied in [24]), that the typical or average correlation coefficient between any two shadowing terms $S_{i}$ would approach 1 as $d$ increases. 


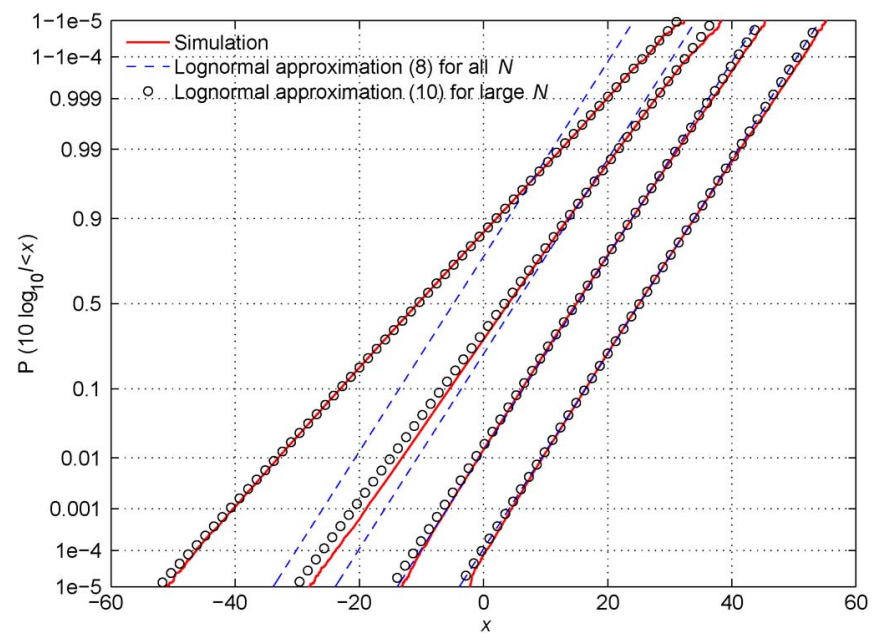

Fig. 5. Distribution of the total interference power $I$ as plotted on "lognormal paper" [41]. The interference is measured at a distance of $d=2 \mathrm{~km}$ from the center of the interferer field, with $N=1,10,100,1000$ (from left to right).

We also observe that the average relative distance $G_{1}=$ $\mathbb{E}\left\{\ln r_{i}\right\}$ of any interferer $i$ increases with $d$; we can say that $G_{1}$ captures how far, on average, the interferers are from the RX. Similarly, $G_{2}-G_{1}^{2}=\mathbb{V A R}\left\{\ln r_{i}\right\}$ captures the relative spread of the distance between the RX and the average interferer; this quantity decreases with $d$, because the relative spread of $r_{i}$ will indeed decrease with $d$ and tend to 0 as $d \rightarrow \infty$.

In Fig. 4, we observe the effect of the distance between the $\mathrm{RX}$ and a large $(N=500)$ field of interferers. Our analytical result can be said to approximate the cdf of $I$ very accurately for $d \geq 2 \mathrm{~km}$, thus confirming that our approximation requires a cluster layout, but performs poorly when the RX is close to (or indeed, inside [1]) the field of interferers. Our results with other clustered layouts in [25], [26] confirm this.

In Fig. 5, we observe the effect of the number of interferers on the accuracy of our approximation when the layout can be said to be clustered $(d=2 \mathrm{~km})$. For a moderate number of interferers $(N=10)$, the distribution of $I$ is not quite lognormal. However, it is interesting that this trend does not continue: as the number of interferers becomes even larger $(N=100$, 1000 ), the distribution of $I$ again becomes close to lognormal. This behavior is also consistent with simulations in [18] in the case of exchangeable jointly lognormal RVs.

These results are consistent with the literature: indeed, for a small number of correlated terms, the sum of lognormals distribution is not always close to a lognormal [19], [20], [42]. The analysis in [21] indicates that only for certain parameter choices is the sum of many correlated lognormals close to a lognormal. Our simulations in [1] show that even for a large $(N$ up to 10000) number of interferers, the sum interference may not be close to a lognormal in a non-clustered interferer layout. In summary, both the clustered layout and the large number of interferers are required for the lognormal approximation to hold.

The simulations in Figs. 4 and 5, and others in [25], [26] confirm that, for a clustered layout and a large number of interferers, our approximation in (8)-(11) is an accurate method for quickly approximating the distribution of the total interference power.

\section{COncluding Remarks}

We were able to derive a very simple and accurate approximation for the distribution of the total interference coming from a large interferer field, given correlated shadowing and a clustered layout. We found that this distribution is close to a lognormal, with parameters that are easy to compute for any number of interferers.

Our method is well-suited for a large number of interferers, where both Monte Carlo simulation and algorithms for finding the sum of correlated lognormal RVs can be computationally costly, if not prohibitive. It is also of interest that the effect of location-dependent correlation among the shadowing paths can be captured by a single scalar, a fact which may greatly simplify the correlation measurements that may be needed in a particular scenario.

Our approach could be extended to more complex system and channel models. Possible extensions include:

- Have the number of interferers $N$ be a RV (e.g., Poisson distributed), rather than deterministic. This could reflect, for example, interferers being on or off.

- The positions $\left\{\vec{r}_{i}\right\}$ of the interferers need not be independent, but could include more complex statistical behaviors (see [10], [44] for examples).

- The interference powers $\left\{I_{i}\right\}$ in this work are assumed to be long-term averages. A random small-scale fading multiplicative term for each interferer could easily be included.

- Similarly, while we assume all interferer transmit powers to be equal, it would be equally possible to model the transmit powers of the interferers as RVs, with some common distribution based on the transmission protocols used.

- It is also quite simple to model a directional receive antenna at the RX, simply by weighing the distribution of the interferers by a function of the angle of arrival. Our method may in fact be very well-suited for such a scenario: even if the RX is not in a clustered layout of interferers, the fairly narrow beam of its directional antenna would receive interference primarily from one direction, and our approximation could still apply.

- It may be useful to study the total interference as a random process in time, particularly in mobility scenarios. Several models are available for describing the auto-correlation function of the shadowing on a mobile link [24], [39], [40], [45].

In [26], we describe in more detail how these extensions could be made. In most cases exchangeability is maintained, and our cross-moment matching analysis can be applied, with minor modifications.

\section{APPENDiX A}

In [18] we have given a proof based on a more complicated decomposition. Here, we give a simpler but essentially similar proof, inspired by the decomposition in [45]. The more general result in [21] also independently gives a similar proof. 
Proof: Let $Z, Z_{1}, \ldots, Z_{N}$ be iid standard Gaussian RVs. Then we can write

$$
V_{i}=\mu+\sigma \sqrt{\rho} Z+\sigma \sqrt{1-\rho} Z_{i}, \quad \forall i
$$

to obtain the same statistical distribution for $V_{i}$ as described in Section III-A. This decomposition is along the lines of Theorem 1, where we see that the exchangeable set $\left\{V_{i}\right\}$ may be written as a function of one common term $Z$, and of individual iid terms $Z_{i}$. From (5), we have

$$
V / N=e^{\mu+\sigma \sqrt{\rho} Z} \cdot \frac{1}{N} \sum_{i=1}^{N} e^{\sigma \sqrt{1-\rho} Z_{i}} .
$$

Now, from the Weak Law of Large Numbers [31], we have

$$
\frac{1}{N} \sum_{i=1}^{N} e^{\sigma \sqrt{1-\rho} Z_{i}} \stackrel{\mathcal{D}}{\longrightarrow} e^{(1-\rho) \sigma^{2} / 2},
$$

a constant. We may then apply Slutsky's theorem [31] to obtain

$$
V / N \stackrel{\mathcal{D}}{\longrightarrow} e^{\mu+(1-\rho) \sigma^{2} / 2+\sigma \sqrt{\rho} Z},
$$

which is a lognormal RV with parameters given by (6).

\section{APPENDiX B}

Here, we derive the correspondence between the parameters of the SEJLN problem and our interference problem. We first set the common factor $K$ to 1 to simplify the expressions.

We approximate the statistics of $\left\{\ln I_{i}\right\}$ with those of $\left\{V_{i}\right\}$ by matching the cross-moments

$$
\begin{aligned}
\mathbb{E}\left\{V_{i}\right\} & =\mu, \\
\mathbb{E}\left\{V_{i}^{2}\right\} & =\sigma^{2}+\mu^{2}, \\
\mathbb{E}\left\{V_{i} V_{j}\right\} & =\rho \sigma^{2}+\mu^{2}, i \neq j,
\end{aligned}
$$

with the corresponding respective cross-moments

$$
\begin{aligned}
\mathbb{E}\left\{\ln I_{i}\right\}= & \mathbb{E}\left\{\lambda S_{i}-\beta \ln r_{i}\right\}=-\beta G_{1}, \\
\mathbb{E}\left\{\ln ^{2} I_{i}\right\}= & \mathbb{E}\left\{\lambda^{2} S_{i}^{2}+\beta^{2} \ln ^{2} r_{i}-2 \beta \lambda S_{i} \ln r_{i}\right\} \\
= & \lambda^{2} \sigma_{\mathrm{s}}^{2}+\beta^{2} G_{2}, \\
\mathbb{E}\left\{\ln I_{i} \ln I_{j}\right\}= & \mathbb{E}\left\{\lambda^{2} S_{i} S_{j}+\beta^{2} \ln r_{i} \ln r_{j}\right\} \\
& -\beta \lambda \mathbb{E}\left\{S_{j} \ln r_{i}+S_{i} \ln r_{j}\right\} \\
= & \lambda^{2} \sigma_{\mathrm{s}}^{2} G_{\text {cor }}+\beta^{2} G_{1}^{2}, i \neq j,
\end{aligned}
$$

where

$$
\begin{aligned}
G_{n} & =\mathbb{E}\left\{\ln ^{n} r_{i}\right\} \text { and } \\
G_{\text {cor }} & =\frac{\mathbb{E}\left\{S_{i} S_{j}\right\}}{\sigma_{\mathrm{s}}^{2}}, i \neq j
\end{aligned}
$$

are quantities that need to be evaluated numerically in general.
We now equate (19) with (20), and reintroduce the factor $K$, which is a common scaling factor that simply modifies the logarithmic mean of the lognormal RV:

$$
\begin{aligned}
\mu & =\ln K-\beta G_{1}, \\
\sigma^{2} & =\beta^{2}\left(G_{2}-G_{1}^{2}\right)+\lambda^{2} \sigma_{\mathrm{s}}^{2}, \\
\rho & =\frac{\lambda^{2} \sigma_{\mathrm{s}}^{2}}{\sigma^{2}} G_{\mathrm{cor}} .
\end{aligned}
$$

We substitute these into (7) and, by equating $\left(m_{I}, s_{I}^{2}\right)=$ $\left(m_{V}, s_{V}^{2}\right)$, we obtain the solution (8).

\section{REFERENCES}

[1] S. S. Szyszkowicz, F. Alaca, H. Yanikomeroglu, and J. S. Thompson, "Aggregate interference distribution from large wireless networks with correlated shadowing: An analytical-numerical-simulation approach," IEEE Trans. Veh. Technol., vol. 60, no. 6, pp. 2752-2764, Jul. 2011.

[2] B. Hagerman, "Downlink relative co-channel interference powers in cellular radio systems," in Proc. IEEE Veh. Technol. Conf., Jul. 1995, vol. 1, pp. $366-370$

[3] W. Mohr, "Heterogeneous networks to support user needs with major challenges for new wideband access systems," Wireless Pers. Commun., vol. 22, no. 2, pp. 109-137, Aug. 2002.

[4] F. Berggren, "An error bound for moment matching methods of lognormal sum distributions," Eur. Trans. Telecommun., vol. 16, no. 6, pp. 573-577, Nov./Dec. 2005.

[5] M. H. Ismail and M. M. Matalgah, "Outage probability analysis in cellular systems with noisy Weibull-faded lognormal-shadowed links," in Proc. IEEE Symp. Comput. Commun., Jun. 2005, pp. 269-274.

[6] J. Salo, L. Vuokko, H. M. El-Sallabi, and P. Vainikainen, "An additive model as a physical basis for shadow fading," IEEE Trans. Veh. Technol., vol. 56, no. 1, pp. 13-26, Jan. 2007.

[7] M. Win, P. Pinto, and L. Shepp, "A mathematical theory of network interference and its applications," Proc. IEEE, vol. 97, no. 2, pp. 205230, Feb. 2009.

[8] M. Aljuaid and H. Yanikomeroglu, "Investigating the Gaussian convergence of the distribution of the aggregate interference power in large wireless networks," IEEE Trans. Veh. Technol., vol. 59, no. 9, pp. 44184424, Nov. 2010.

[9] R. Hekmat and P. V. Mieghem, "Interference power sum with log-normal components in ad-hoc and sensor networks," in Proc. Int. Symp. Model. Optim. Mobile, Ad Hoc, Wireless Netw., Apr. 2005, pp. 174-182.

[10] R. Ganti and M. Haenggi, "Interference and outage in clustered wireless ad hoc networks," IEEE Trans. Inf. Theory, vol. 55, no. 9, pp. 4067-4086, Sep. 2009.

[11] K. Gulati, B. L. Evans, J. G. Andrews, and K. R. Tinsley, "Statistics of cochannel interference in a field of Poisson and Poisson-Poisson clustered interferers," IEEE Trans. Signal Process., vol. 58, no. 12, pp. 6207-6222, Dec. 2010.

[12] Y. Zhang et al., "Home M2M networks: Architectures, standards, and QoS improvement," IEEE Commun. Mag., vol. 49, no. 4, pp. 44-52, Apr. 2011.

[13] Cisco Systems, Inc., 20 Myths of Wi-Fi Interference: Dispel Myths to Gain High-Performing and Reliable Wireless, San Jose, CA, USA 2007, white Paper.

[14] Z. Horvath and D. Varga, "Elimination of RLAN interference on weather radars by channel allocation in $5 \mathrm{GHz}$ band," in Proc. Int. Conf. Ultra Modern Telecommun. Workshops, Oct. 2009, pp. 1-6.

[15] K. Koufos, K. Ruttik, and R. Jantti, "Aggregate interference from WLAN in the TV white space by using terrain-based channel model," in Proc. 7th Int. ICST Conf. Cogn. Radio Oriented Wireless Netw. Commun., Jun. 2012, pp. 185-189.

[16] A. Safak and R. Prasad, "Effects of correlated shadowing signals on channel reuse in mobile radio systems," IEEE Trans. Veh. Technol., vol. 40, no. 4, pp. 708-713, Nov. 1991.

[17] A. A. Abu-Dayya and N. C. Beaulieu, "Outage probabilities in the presence of correlated lognormal interferers," IEEE Trans. Veh. Technol., vol. 43, no. 1, pp. 164-173, Feb. 1994.

[18] S. S. Szyszkowicz and H. Yanikomeroglu, "Limit theorem on the sum of identically distributed equally and positively correlated joint lognormals," IEEE Trans. Commun., vol. 57, no. 12, pp. 3538-3542, Dec. 2009. 
[19] M. Di Renzo, L. Imbriglio, F. Graziosi, and F. Santucci, "Smolyak's algorithm: A simple and accurate framework for the analysis of correlated log-normal power-sums," IEEE Commun. Lett., vol. 13, no. 9, pp. 673675, Sep. 2009.

[20] A. Hasan Mahmoud, "New quadrature-based approximations for the characteristic function and the distribution function of sums of lognormal random variables," IEEE Trans. Veh. Technol., vol. 59, no. 7, pp. 33643372, Sep. 2010.

[21] N. C. Beaulieu, "An extended limit theorem for correlated lognormal sums," IEEE Trans. Commun., vol. 60, no. 1, pp. 23-26, Jan. 2012.

[22] M. Abdel-Hafez, "Co-channel interference probability of cellular mobile radio in generalized fading and correlated shadowing environment," in Proc. Commun. Syst. Netw., Sep. 2002, pp. 298-303.

[23] S. Roy and S. Kundu, "Outage analysis in presence of correlated interferers in a cognitive-cellular network," in Proc. IEEE Symp. Ind. Electron. Appl., Oct. 2010, pp. 100-105.

[24] S. S. Szyszkowicz, H. Yanikomeroglu, and J. S. Thompson, "On the feasibility of wireless shadowing correlation models," IEEE Trans. Veh. Technol., vol. 59, no. 9, pp. 4222-4236, Nov. 2010

[25] S. S. Szyszkowicz and H. Yanikomeroglu, "Analysis of interference from large clusters as modeled by the sum of many correlated lognormals," in Proc. IEEE Wireless Commun. Netw. Conf., Mar./Apr. 2008, pp. 741-745.

[26] S. S. Szyszkowicz, "Interference from large wireless networks under correlated shadowing," Ph.D. dissertation, Carleton University, Ottawa, ON, Canada, Jan., 2011.

[27] B. de Finetti, "La prévision: Ses lois logiques, ses sources subjectives," Ann. l'institut Henri Poincaré, vol. 7, no. 1, pp. 1-68, 1937.

[28] B. de Finetti, Theory of Probability: A Critical Introductory Treatment. Hoboken, NJ, USA: Wiley, 1970.

[29] R. L. Taylor, P. Z. Daffer, and R. F. Patterson, Limit Theorems for Sums of Exchangeable Random Variables. Totowa, NJ, USA: Rowman \& Allanheld, 1985

[30] E. Hewitt and L. J. Savage, "Symmetric measures on Cartesian products," Trans. Amer. Math. Soc., vol. 80, no. 2, pp. 470-501, Nov. 1955.

[31] V. K. Rohatgi and A. K. M. Ehsanes Saleh, An Introduction to Probability and Statistics, 2nd ed. Hoboken, NJ, USA: Wiley-Interscience, 2001.

[32] A. J. Viterbi, A. M. Viterbi, and E. Zehavi, "Other-cell interference in cellular power-controlled CDMA," IEEE Trans. Commun., vol. 42, no. 234, pp. 1501-1504, Feb.-Apr. 1994.

[33] M. Chiani, A. Conti, and O. Andrisano, "Outage evaluation for slow frequency-hopping mobile radio systems," IEEE Trans. Commun., vol. 47, no. 12, pp. 1865-1874, Dec. 1999.

[34] S. S. Szyszkowicz, H. Yanikomeroglu, E. Fituri, and S. Periyalwar, "Analytical modeling of interference in cellular fixed relay networks," in Proc. IEEE Can. Conf. Elect. Comput. Eng., May 2006, pp. 1562-1565.

[35] P. Harley, "Short distance attenuation measurements at $900 \mathrm{MHz}$ and $1.8 \mathrm{GHz}$ using low antenna heights for microcells," IEEE J. Sel. Areas Commun., vol. 7, no. 1, pp. 5-11, Jan. 1989.

[36] D. Kitchener et al., Correlated Lognormal Shadowing Model, Jul. 2006, IEEE 802.16 Session 44, Tech. Rep.

[37] J. F. Monserrat, R. Fraile, and L. Rubio, "Application of alternating projection method to ensure feasibility of shadowing cross-correlation models," IET Electron. Lett., vol. 43, no. 13, pp. 724-725, Jun. 2007.

[38] T. Klingenbrunn and P. Mogensen, "Modelling cross-correlated shadowing in network simulations," in Proc. IEEE Veh. Technol. Conf., Sep. 1999, vol. 3, pp. 1407-1411.

[39] M. Gudmundson, "Correlation model for shadow fading in mobile radio systems," IEE Electron. Lett., vol. 27, no. 23, pp. 2145-2146, Nov. 1991.

[40] H. Arnold, D. Cox, and R. Murray, "Macroscopic diversity performance measured in the $800-\mathrm{MHz}$ portable radio communications environment," IEEE Trans. Antennas Propag., vol. 36, no. 2, pp. 277-281, Feb. 1988.

[41] N. Beaulieu and Q. Xie, "An optimal lognormal approximation to lognormal sum distributions," IEEE Trans. Veh. Technol., vol. 53, no. 2, pp. 479-489, Mar. 2004.

[42] N. B. Mehta, J. Wu, A. F. Molisch, and J. Zhang, "Approximating a sum of random variables with a lognormal," IEEE Trans. Wireless Commun., vol. 6, no. 7, pp. 2690-2699, Jul. 2007.
[43] B. Alkire, "Cholesky Factorization of Augmented Positive Definite Matrices," Elect. Eng. Dept., UCLA, Los Angeles, CA, USA, Tech. Rep., Dec. 2002.

[44] U. Schilcher, M. Gyarmati, C. Bettstetter, Y. W. Chung, and Y. H. Kim, "Measuring inhomogeneity in spatial distributions," in Proc. IEEE Veh. Technol. Conf., May 2008, pp. 2690-2694.

[45] R. Fraile, J. F. Monserrat, J. Gozalvez, and N. Cardona, "Mobile radio bi-dimensional large-scale fading modelling with site-to-site crosscorrelation," Eur. Trans. Telecommun., vol. 19, no. 1, pp. 101-106, Jan./Feb. 2008.

Sebastian S. Szyszkowicz (S'06-M'11) received the B.A.Sc. degree in electrical engineering (specializing in communications) from the University of Ottawa, ON, Canada, in 2003, and the M.A.Sc. and Ph.D. degrees in electrical and computer engineering from Carleton University, Ottawa in 2007 and 2011, respectively. In winter 2008, he was a visiting Researcher at the University of Edinburgh, Edinburgh, U.K., and has given several invited talks about the Ph.D. research at universities in Europe and Canada. In 2011-2012, he was a visiting fellow at the Communications Research Centre (CRC) Canada. Since 2013, he has been a postdoctoral fellow at the University of Ottawa, Canada. His research focuses on large-scale wireless interference and propagation.

$\mathrm{He}$ is the recipient of research awards from the Natural Sciences and Engineering Research Council of Canada (NSERC): Postgraduate Scholarships (PGS) at both the Masters and the Ph.D. levels, and Visting Fellow (VF) and Postdoctoral Fellow (PDF) at the postdoctoral level.

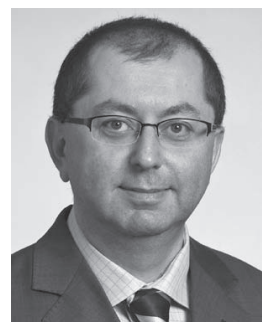

Halim Yanikomeroglu (S'96-M'98-SM'12) was born in Giresun, Turkey, in 1968. He received the B.Sc. degree in electrical and electronics engineering from the Middle East Technical University, Ankara, Turkey, in 1990, and the M.A.Sc. degree in electrical engineering (now ECE) and the Ph.D. degree in electrical and computer engineering from the University of Toronto, ON, Canada, in 1992 and 1998, respectively.

During 1993-1994, he was with the R\&D Group of Marconi Kominikasyon A.S., Ankara, Turkey. Since 1998 he has been with the Department of Systems and Computer Engineering at Carleton University, Ottawa, where he is now a Full Professor. His research interests cover many aspects of wireless technologies with a special emphasis on cellular networks. He coauthored about 60 IEEE journal papers, and has given a high number of tutorials and invited talks on wireless technologies in the leading international conferences. In recent years, his research has been funded by Huawei, Blackberry, Samsung, Communications Research Centre of Canada (CRC), Telus, and Nortel. This collaborative research resulted in about 18 patents (granted and applied). Dr. Yanikomeroglu has been involved in the organization of the IEEE Wireless Communications and Networking Conference (WCNC) from its inception, including serving as Steering Committee Member as well as the Technical Program Chair or Co-Chair of WCNC 2004, WCNC 2008, and WCNC 2014 to be held in Istanbul. He was the General Co-Chair of the IEEE Vehicular Technology Conference Fall 2010 held in Ottawa. He has served in the editorial boards of the IEEE TRANSACTIONS ON COMMUNICATIONS, IEEE TRANSACTIONS ON WIRELESS COMMUNICATIONS, and IEEE COMMUNICATIONS SURVEYS \& Tutorials. He was the Chair of the IEEE's Technical Committee on Personal Communications (now called Wireless Technical Committee). He is a Distinguished Lecturer for the IEEE Vehicular Technology Society.

Dr. Yanikomeroglu is a recipient of the Carleton University Faculty Graduate Mentoring Award in 2010, the Carleton University Graduate Students Association Excellence Award in Graduate Teaching in 2010, and the Carleton University Research Achievement Award in 2009. Dr. Yanikomeroglu spent the 2011-2012 academic year at TOBB University of Economics and Technology, Ankara, Turkey, as a Visiting Professor. He is a registered Professional Engineer in the province of Ontario, Canada. 